\section{The Young Faculty Meeting 2021 - A Focus on Group Management}

\section{Jonathan de Roo*, Nina Hartrampf*, and Leo Merz* \\ ${ }^{*}$ Correspondence: Prof. Dr. J. De Roo, E-mail: jonathan.deroo@unibas.ch, University of Basel, Department of Chemistry, Mattenstrasse 24a, BPR 1096, CH-4058 Basel, Switzerland; Prof. Dr. N. Hartrampf, E-mail: nina.hartrampf@ chem.uzh.ch, University of Zurich, Department of Chemistry, Winterthurerstrasse 190, CH-8057 Zurich, Switzerland; Dr. L. Merz, E-mail: chemistry@scnat.ch, Swiss Academy of Sciences SCNAT, Platform Chemistry, Laupenstr. 7, CH-3008 Bern, Switzerland}

The 2021 edition of the Young Faculty Meeting surmounted unusual challenges: due to surges in COVID cases, the meeting was moved from winter to early summer. Exceptional times require exceptional measures and the event was not held at the usual location, the 'Haus der Universität', but on the Gurten hill, twenty minutes outside Bern. This fantastic location allowed for a flexible meeting, having both an outside and an inside stage with a set of COVID precautionary measures for each. In the true spirit of the YFM, we could not accept a virtual conference and thus the participants enjoyed their first in-person meeting in more than one year.

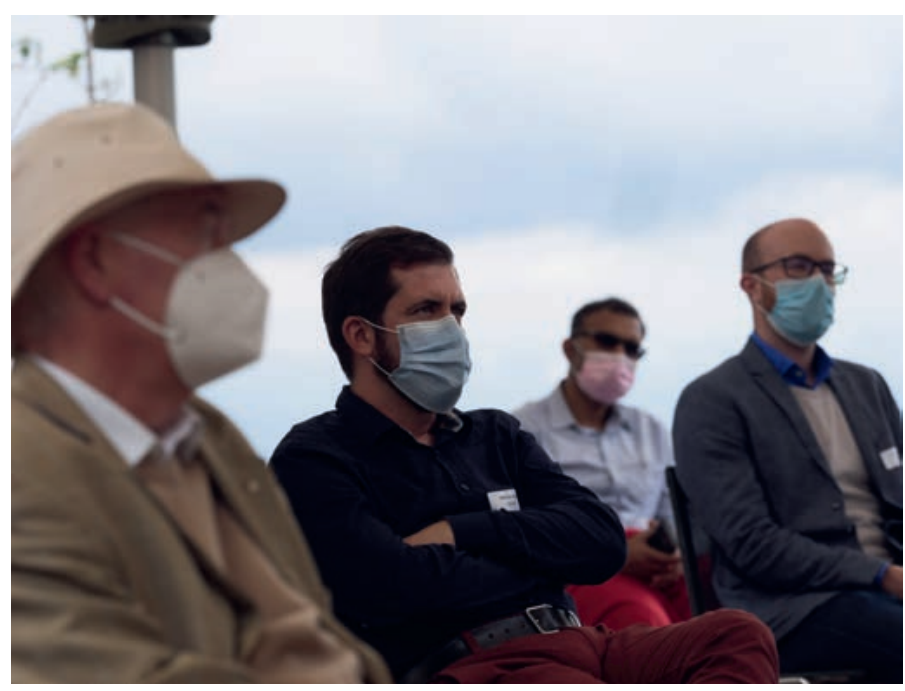

Speakers and audience showed great flexibility for the unconventional setting: outdoors and changes to indoor for some talks.

The first half of the meeting was dedicated to giving young PIs a platform to present their research to their peers, promoting visibility and fostering interdisciplinary collaboration. Kumar Varoon Agrawal (EPFL) is interested in improving the efficiency of molecular separation and introduced his membrane-based separation techniques. These membranes are built up either from monolayer thick graphene or exfoliated zeolites. For example, he discussed the challenges of separating $\mathrm{CO}_{2}$ from $\mathrm{N}_{2}$, which are only $0.34 \AA$ different. Precise control of pore size in graphene is thus required, and was achieved by treatments of graphene with ozone where kinetics of nucleation and growth was controlled. Michel Rickhaus (University of Zurich) gave a TED-like talk about molecular shape. Function follows form, is his catchphrase. Thinking conceptually about the assembly of molecules, a linear stack of flat molecules seems the simplest example, and properties can be transferred through this ordered structure. However, this stack is prone to translational and rotational disorder. Saddle shaped graphene-like sheets exactly prohibit both translation and rotation and are actively synthesized and analyzed in the Rickhaus Lab. Fabienne Schwab (AMI/University of Fribourg) showed how biodegradable silica nanoagrochemicals can be used to strengthen the inner defenses of plants. Instead of killing the pests directly and thus risking collateral damage in the ecosystem, the nanoparticles rally the immune system of the plants. This strategy could avoid the need to continuously increase the dosage of agrochemicals, since in the classical strategy, the pests start to develop resistance to the agrochemicals. Michal Shoshan (University of Zurich) managed to captivate the audience with a courageous talk without any visual aids. She talked about the four most dangerous metals for humans: cadmium, arsenic, mercury and lead. Of these four, lead poses the most significant problem due to its abundance in our environment (lead-based paint, leaded fuel, lead in water pipes, etc.). More than one million deaths per year are caused by lead globally, and every third child suffers from lead poisoning. The Shoshan lab uses peptides as a scaffold to develop heavy metal chelating agents. Peptides are ideal for a combinatorial screening (e.g. genetically-encoded peptide libraries by directed evolution) to optimize the metalbinding properties of the scavenger. The group strives to develop next-generation medicinal and environmental solutions against this poisonous metal. Maksym Yarema (ETHZ) talked about the beauty of inorganic complexity in colloidal nanocrystals. Starting from ultra-monodisperse metal nanocrystals as seeds, intermetallic nanocrystals can be synthesized using a liquid metal precursor. High control over size and composition is achieved. This strategy also avoids the traditional difficulties of different redox potentials when synthesizing intermetallic nanoparticles. Furthermore, from highly complex stoichiometries, order may arise from chaos. For example, $\mathrm{Cu}_{5} \mathrm{Zn}_{2} \mathrm{In}_{11} \mathrm{Se}_{21}$ and $\mathrm{Cu}_{3} \mathrm{In}_{5} \mathrm{Se}_{9}$ nanocrystals have optimized compositions where the cationic vacancies are perfectly ordered, and the nanocrystals are highly emissive in the visible region. Bruno Correia (EPFL) inspired the audience with computationally-guided protein design. He distinguishes a traditional top-down approach, where natural proteins are adapted to a different function, from a bottom-up approach, where a new protein is built around a particular functionality. The Correia group is using the latter strategy and has developed computational tools to assemble proteins in a de novo fashion. A second research thrust of his group is mapping the surface of proteins. Much like face recognition software can identify humans, the Correia group uses pattern recognition algorithms to classify protein surfaces. This tool is then used to predict binding partners involved in protein-protein interactions.

Although the weather frequently changed from sunshine to rain, which led to multiple changes of location, the overall atmosphere in the morning was relaxed and the participants enjoyed the scientific presentations and the face-to-distanced-face con- 


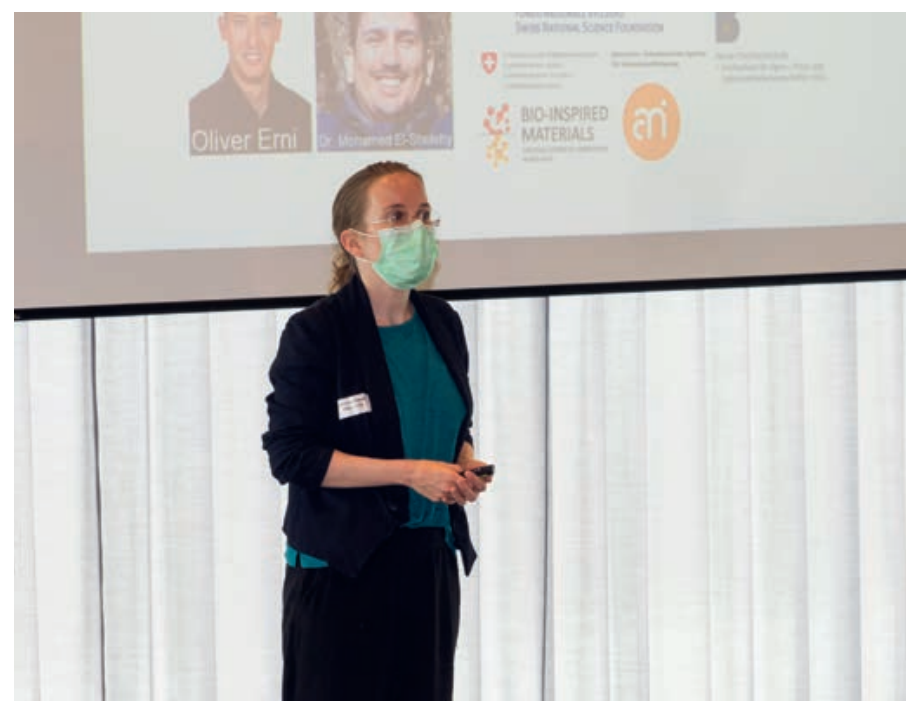

Fabienne Schwab presenting her research on biodegradable nanoagrochemicals - on the indoor stage.

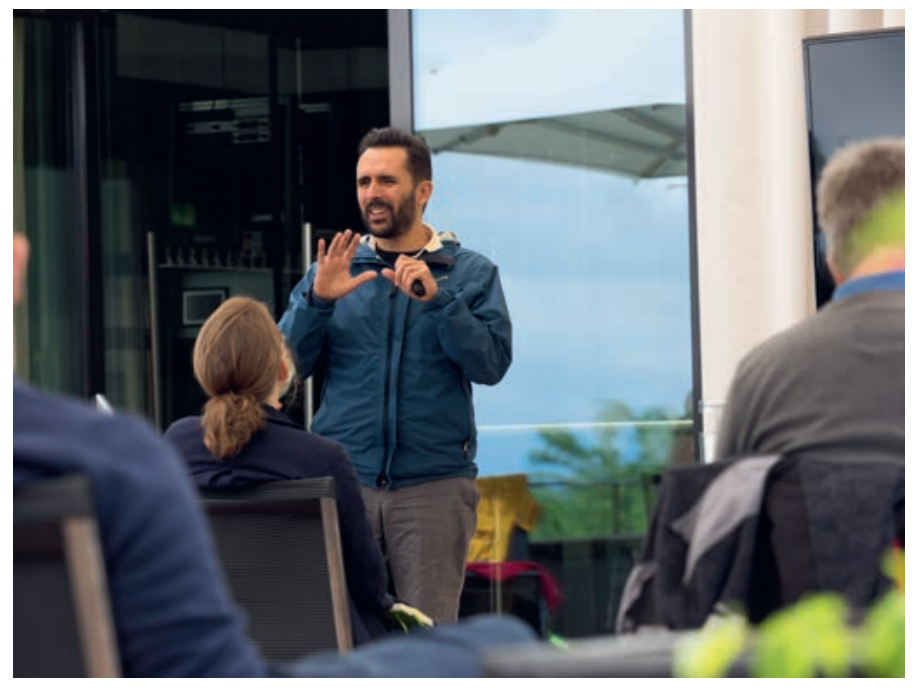

Bruno Correia presenting computationally-guided protein design in the outdoor setting.

versations during the coffee and lunch breaks.

The second half of the meeting was dedicated to aspects around group management. $\mathrm{PhD}$ students and postdocs receive extensive education in conducting research, but are usually taught very little about being a leader or a manager. To start closing this gap, we chose 'group management' - which for us includes hiring, group culture, evaluation, feedback, mental health, data management and more - as the topic of this year's YFM. First, Matthias Töwe (ETHZ, ETH Library) discussed the importance of data management. All the data from a lab should be managed, if even the managerial decision is to classify the data 'of no further interest/to be shelved'. The data that is retained should be FAIR, meaning it should be Findable, Accessible, Interoperable

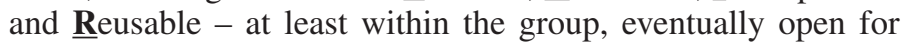
others. In addition, more and more funders (like SNSF) require that the published data is open, being thus freely accessible via open repositories, ready for immediate reuse or reevaluation by other researchers. Furthermore, Matthias encouraged the young faculty to have a discussion about data in their research community. What do you and your colleagues expect from each other? A discussion about data management should not be a burden enforced by the administrators, but an opportunity of every field to become more reproducible and to accelerate discovery. Maarten
Van Winckel (University of Basel, innovation office) showed how certain management tools from industry can be translated to a research context. KPI's (Key Performance Indicators) allow to establish a communication line between advisor and $\mathrm{PhD}$ student to discuss expectations and workload management. This is a critical issue since $\mathrm{PhD}$ students have significantly more mental health problems compared to the average population. One in three students struggles with signs of depression. These KPIs are preferably SMART, which means they need to be Specific, Measurable, Achievable, Relevant, and Time-based. It is recommended to not only set KPIs for the individuals, but also one for the group as a whole (e.g. being the safest lab, or the most sustainable lab). A personal KPI, focusing on personal development of the student, is also crucial to prepare the students for their career after the $\mathrm{PhD}$. It is the duty of PIs as good managers and leaders to focus not only on the next research paper but also on the personal growth of their students and postdocs. Our current $\mathrm{PhD}$ students are the leaders of tomorrow, not just in academia but in society as a whole.

Finally, the afternoon was closed by a panel discussion on group management. As panel members, we invited Karl Gademann (University of Zurich), Alain de Mesmaeker (previously Syngenta), Maarten Van Winckel, and Madeleine Wirz (Sympany). It was a lively discussion on hiring, setting group culture, evaluation/constructive feedback, interpersonal interactions, and student mental health. According to our four panel members the key to good management is: (i) foster creativity and independence, (ii) lead by example, (iii) enable others to live their best life, and (iv) stay curious and modest. Regarding the topic of hiring, certain panel members preferred personality over skill sets. It was reasoned that one can teach a person scientific techniques, but changing behavior or an attitude is more difficult. A quote from a panel member: "hire for personality, train for skills". A panel member also suggested to challenge candidates with very difficult tasks or constructive criticism in the (second) interview and see how they cope with it. Do remain open or do they react defensively? Following up on the topic of specific interview questions and techniques, we were also wondering about favorite interview questions and here are some examples:

- What do you bring to the group?

- What is your favorite experiment? What excites you?

- Tell me all the best things in your life? (let them speak)

- Do you have questions for me?

A lively discussion arose around the suggestion to request certain tasks from a candidate such as reading a paper, writing a one-page summary or proposal, once the preselection process is done. It was concluded that a time commitment of one day for a job interview is not excessive. In return, be prepared to give more detailed feedback. In any case, make the interview process clear and transparent. For many of your candidates, this is their first job interview and they have no idea what to expect. Hiring is the sole responsibility of the PI but it was discussed that the group should be involved at some point and a group's veto to not hire a candidate should be respected. Furthermore, it is recommended to build a strong network among peers to receive trustworthy recommendations regarding potential candidates. Regarding group culture, it was emphasized by a panel member that a critical aspect is to establish psychological safety, where every group member is heard equally and can voice their opinions without fear of repercussions. Gossip should be avoided at all times, i.e. where a PI talks bad about one group member to another group member. It was recommended to celebrate the 'onboarding' of a new group member. Do not simply throw a new person into the group, but take time to make this a special moment. Regarding evaluation and feedback, the system of Key Performance Indicators was re-emphasized to open these communication lines and track 
progress. Despite all these efforts, it is of course still possible that a group member does not function well in the research environment. The ultimate resort, ending a contract, causes typically great anxiety among the remaining group members. However, it can be necessary to remove a toxic person from the group. When the communication is clear and the case is severe, the group will eventually (maybe not immediately) realize it was a good decision to let this person go. Even highly experienced managers lose sleep over firing a group member and this reaction is totally normal for a PI, too. Regarding the relation between PI and group; there is a quote from Peter F. Drucker: "As a manager you're paid to be uncomfortable. If you're comfortable, it's a sure sign you're doing things wrong". PIs should keep a lot to themselves and not burden the group with their troubles. Often a PI needs to make a difficult decision and stick to it. This does not mean a PI cannot have open and transparent communication with the group. Even feedback from the group about the PI's leadership style should be on the table. Finally, student mental health is a problem to take serious. Be aware of signs of depression (the KPIs can also be an early warning system). However, it is not the PIs position to become a psychiatrist. Early detection of a problem, and referral to a professional is key. Asking the question "how are you doing" and being interested in the answer is a great start.

We took away from the afternoon that this group of young PIs care a lot about the wellbeing of their students and post-docs, and not just about their research results. It was mentioned by the participants that it is great to realize that more people are in the same situation and struggle with the same issues on management. This young faculty meeting certainly built a strong support network where the young faculty left the meeting with the feeling that there are other like-minded people to fall back on and to ask for advice.

Received: July 6, 2021 